\title{
CDR Assessment with Superpixel Segmentation an Effort towards Automatic Glaucoma Detection
}

\author{
Manisha joshi ${ }^{1}$, Prof. V.S.Bhatlawande ${ }^{2}$ \\ ${ }^{1}$ Electronics \& Telecommunication SCOE, Sudumbare Pune, India \\ ${ }^{2}$ Electronics \& Telecommunication SCOE, Sudumbare Pune, India
}

\begin{abstract}
:
Objective: Glaucoma is a chronic eye defect that causes parmanent loss of vision.it is second major cause behind blindness.in this desease optic nerve slowly gets damaged .In early stages of glaucoma patient has no symptoms and no pain.by the time glaucoma affects the side or peripheral visions causing partial blindness, and will turn into complete blindness if not dignosed with in time.because the effect is complete irrversible. Cup to disc ratio is the useful in glaucoma screening. In this paper we offer a novel method for CDR assessment that is superpixel segmentation .SLIC algoritham is used for superpixel generation. In the praposed method optic disc is first segmented and reconstructed using superpixel classification
\end{abstract}

Key words: CDR -cup to disc ratio,SLIC,glaucoma screening superpixel segmentation.

\section{INTRODUCTION}

Glaucoma is a chronic eye desease which is irreversible.It is a slow process with no pain no symptom once neglected can cause partial or complete blindness.In this the opical nerve head gets damaged,causing defective peripheral vision Slowly reaching towards centre.it is second leading cause of blindness.according to the survey reports $80 \%$ of the population will be affected by time 2020 .as it has no indications glaucoma is also called as the sient theft of eyesight.detection in time is very important ,which helps in reducing the speed of process. Earlier there was no effective method for glaucoma screening over a large population. The IOP(intraocular pressure ) measurement,visual field test and $\mathrm{ONH}$ (optic nerve head) assesment are the methods with which opticians and lab experts are familier with.but these method also has some drawbacks.IOP measurement has low accuracy in glaucoma detection.visual field test requires the specialized equipments and experties.so these two are not feseble for large population glaucoma screening. ONH assesment is the promising method but manualy it is time consuming. Cup to disc ratio is receiving much attention now a days.but again there is problem of 3D retina images such as stereo images and OCT optical coherance tomography.but cost is raised.so can not be forded for large population glaucoma screening. As an alternative 2D fundus images with additional hardware /software made our objective worh a experiment

\section{II.SLIC ALGORITHM FOR SUPERPIXEL SEGMENTATION}

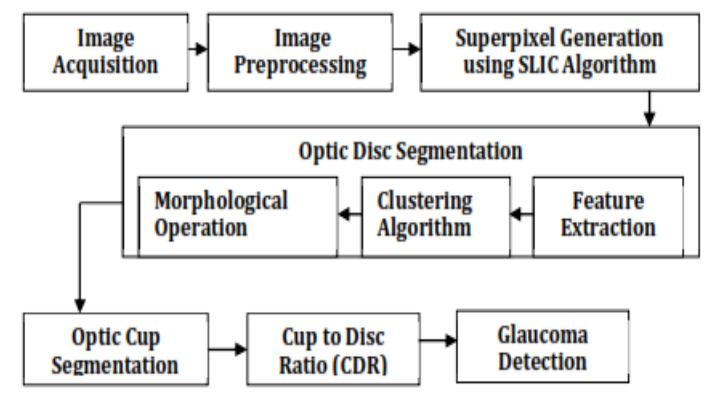

Fig 1. Block Diagram of Proposed System

Superpixels captures redundancy of intensity value to compute local image features for the segmentation. Superpixel image segmentation is faster, easy to use, and produce high quality segmentation but still they often suffer from a high computational cost, poor quality segmentation, inconsistent size and shape, or contain multiple difficult-to-tune parameters. Superpixel segmentation technique is widely used for object class recognition and medical image segmentation because of its simplicity and greater performance at a lower computational cost in comparison to existing methods. We have used simple linear iterative clustering (SLIC) algorithm to perform a local clustering of pixels in the 5-D space defined by the L, $\mathrm{a}, \mathrm{b}$ values of the CIELAB color space and the $\mathrm{x}, \mathrm{y}$ pixel coordinates. A novel distance measure enforces compactness and regularity in the superpixel shapes, and seamlessly accomodates grayscale as well as color images SLIC generates superpixels by 
clustering pixels based on their color similarity and proximity in the image plane which can be done by five-dimensional [labxy] space, where [lab] is the pixel color vector in CIELAB color space, which is widely considered as perceptually uniform for small color distances, and xy is the pixel position. For an image with $\mathrm{N}$ pixels, the approximate size of each superpixel is therefore $\mathrm{N} / \mathrm{K}$ pixels, where $\mathrm{K}$ is total number of superpixels. For roughly equally sized superpixels there would be a superpixel center at every grid interval $S=p \mathrm{~N} / \mathrm{K}$. At the onset of ouralgorithm, we choose $\mathrm{K}$ superpixel cluster centers $\mathrm{Ck}=[\mathrm{lk}, \mathrm{ak}, \mathrm{bk}, \mathrm{xk}, \mathrm{yk}] \mathrm{T}$ with $\mathrm{k}=[1, \mathrm{~K}]$ at regular grid intervals $S$. Since the spatial extent of any superpixel is approximately $\mathrm{S}^{2}$ (the approximate area of a superpixel), we can safely assume that pixels that are associated with this cluster center lie within a $2 \mathrm{~S}$ $\times 2 \mathrm{~S}$ area around the superpixel center on the $\mathrm{xy}$ plane. This becomes the search area for the pixels nearest to each cluster center. Euclidean distances in CIELAB color space are perceptually meaningful for small distances (Eq. 1 ). If spatial pixel distances exceed this perceptual color distance limit, then they begin to outweigh pixel color similarities (resulting in superpixels that do not respect region boundaries, only proximity in the image plane). Therefore, instead of using a simple Euclidean norm in the 5D space, we use a distance measure Ds defined as follows:

$$
\begin{gathered}
d_{l a b}=\sqrt{\left(a_{k}-l_{i}\right)^{2}+\left(a_{k}-a_{i}\right)^{2}+\left(b_{k}-b_{i}\right)^{2}} \\
d_{x y}=\sqrt{\left(x_{k}-x_{i}\right)^{2}+\left(y_{k}-y_{i}\right)^{2}} \\
\text { Ds }=d_{l a b}+\frac{m}{s} \cdot d_{x y}
\end{gathered}
$$

where Ds is the sum of the $L^{*} a * b$ distance and the $x y$ plane distance normalized by the grid interval S. A variable $\mathrm{m}$ is introduced in Ds allowing us to control the compactness of a superpixel. The greater the value of $\mathrm{m}$, the more spatial proximity is emphasized and the more compact the cluster. This value can be in the range $[1,20]$. We choose $m=10$ for all the results in this paper. This roughly matches the empirical maximum perceptually meaningful CIELAB distance and offers a good balance between color similarity and spatial proximity.

SLIC begin by sampling K regularly spaced cluster centers and moving them to seed locations corresponding to the lowest gradient position in a $3 \times$ 3 neighborhood. This is done to avoid placing them at an edge and to reduce the chances of choosing a noisy pixel. Image gradients are computed as:

$G(x, y)=\|I(x+1, y)-I(x-1, y)\|^{2}+\| I(x, y+1)$ $I(x, y-1) \|^{2}$

where $\mathrm{I}(\mathrm{x}, \mathrm{y})$ is the lab vector corresponding to the pixel at position (x,y), and $\|$.$\| is the L2 norm. This$ takes into account both color and intensity information. Each pixel in the image is associated with the nearest cluster center whose search area overlaps this pixel. After all the pixels are associated with the nearest cluster center, a new center is computed as the average labxy vector of all the pixels belonging to the cluster. We then iteratively repeat the process of associating pixels with the nearest cluster center and recomputing the cluster center until convergence. At the end of this process, a few stray labels may remain, that is, a few pixels in the vicinity of a larger segment having the same label but not connected to it. While it is rare, this may arise despite the spatial proximity measure since our clustering does not explicitly enforce connectivity. Nevertheless, we enforce connectivity in the last step of our algorithm by relabeling disjoint segments with the labels of the largest neighboring cluster.

\section{SUPERPIXEL SEGMENTATION}

Localization and segmentation of disc are very important in many computer aided diagnosis systems, including glaucoma screening. The localization focuses on finding an disc pixel, very often the centre. It is studied for applications in diabetic screening [13]. Our work focuses on the segmentation problem and the disc is location, The segmentation estimates the disc boundary, which is a challenging task due to blood vessel occlusions, pathological changes around disc, variable imaging conditions, etc. Feature Extraction techniques like clustering algorithm and morphological operations are used for optic disc segmentation. Circular Hough transform is also used to model the disc boundary because of its computational efficiency. we propose a superpixel classification based method and combine it with the deformable model based methods. Superpixels are local, coherent and provide a convenient primitive to compute local image features. They capture redundancy in the image and reduce the complexity of subsequent processing.[16]. In the proposed method, superpixel classification is used for aninitialization of disc boundary and the deformable model is used to fine tune the disc boundary i.e. a superpixel classification based disc initialization for deformable models. The flow chart of the proposed disc segmentation method is summarized in Fig 3. The segmentation consist of a superpixel generation step to divide the image into superpixel, a feature extraction step to compute features from each superpixel, a classification step to determine each superpixel as a disc or non-disc superpixel to estimate the boundary, a deformation step using deformable models to fine tune the disc boundary which is illustrated in fig 2. 


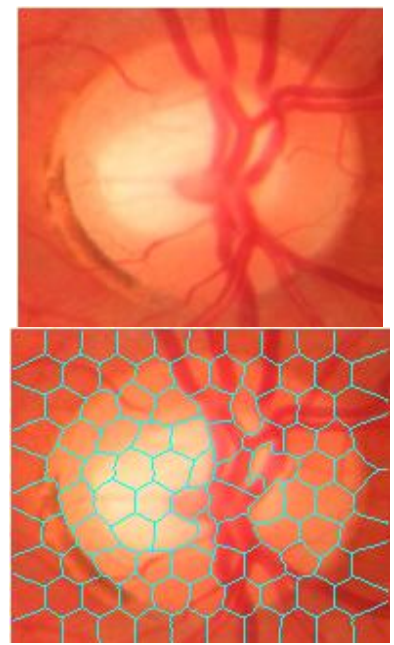

(a)

(b)

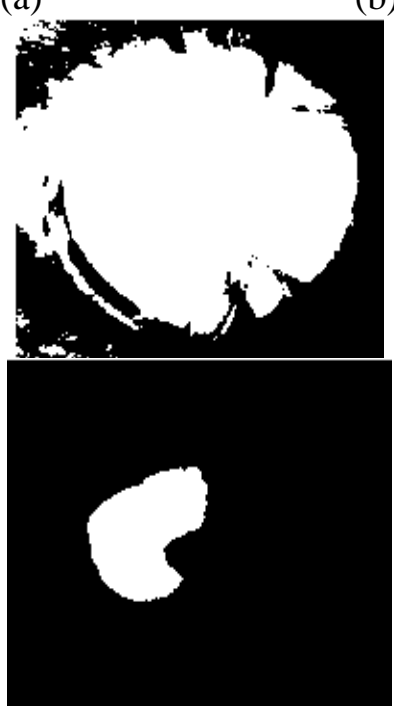

(c)

(d)

Fig. 2 a) original retinal image b) superpixel segmentation c) disc segmentation d) cup segmentation

\section{CONCLUSION}

In this paper, We have proposed the system for glaucoma assessment based on superpixel classification for segmentation of optic disc and optic cup. We have discussed the methods to calculate the CDR from fundus images using segmentation of optic disc and optic cup. We concluded that for detection and diagnosis of glaucoma, firstly, optic disk need to be segmented. After image acquisition, preprocessing is done by applying thresholding, illumination and histogram equalization. The optic disk is segmented using k-means clustering and SLIC algorithm. In future work for diagnosis of glaucoma optic cup need to be segmented. After obtaining Optic Disc \& Optic Cup, CDR is calculated for deciding whether condition of eye is normal or glaucomatous

\section{REFERENCES}

[1]. A. Aquino, M. Gegundez-Arias, and D. Marin, "Detecting the optic disc boundary in digital fundus images using morphological, edge detection, and feature extraction techniques," IEEE Trans. Med. Imag., vol. 29, pp. 1860-1869, 2010.

[2]. A. Hoover and M. Goldbaum, "Locating the optic nerve in a retinal image using the fuzzy convergence of the blood vessels," IEEE Trans. Med. Imag., vol. 22, pp. 951-958, 2003.

[3]. A. P. Rovira and E. Trucco, "Robust optic disc location via combination of weak detectors," in Proc. Int. Conf. IEEE Eng. Med. Bio. Soc., pp. 3542-3545, 2008.

[4]. Centre for Eye Research Australia, Tunnel vision : the economic impact of primary open angle glaucoma. [electronic resource], 2008, http://nla.gov.au/nla.arc-86954.

[5]. D. Michael and O. D. Hancox, "Optic disc size, an important consid-eration in the glaucoma evaluation," Clinical Eye and Vision Care, vol. 11, pp. 59-62, 1999.

[6]. F. Yin, J. Liu, D. W. K. Wong, N. M. Tan, C. Cheung, M. Baskaran, Aung, and T. Y. Wong, "Automated segmentation of optic disc and optic cup in fundus images for glaucoma diagnosis," IEEE Int. Symp. on Computer-Based Medical Systems, pp. 1-6, 2012.

[7]. F. Yin, J. Liu, S. H. Ong, Y. Sun, D. W. K. Wong, N. M. Tan, C. Cheung, M. Baskaran, T. Aung, and T. Y. Wong, "Model-based optic nerve head segmentation on retinal fundus images," Int. Conf. of IEEE Eng. in Med. and Bio. Soc., pp. 2626-2629, 2011.

[8]. G. D. Joshi, J. Sivaswamy, and S. R. Krishnadas, "Optic disk and cup segmentation from monocular color retinal images for glaucoma assessment," IEEE Trans. Med. Imag., vol. 30, pp. 1192-1205, 2011.

[9]. G. D. Joshi, J. Sivaswamy, K. Karan, and R. Krishnadas, "Optic disk and cup boundary detection using regional information," in Proc. IEEE Int. Symp. Biomed. Imag., pp. 948-951, 2010.

[10]. H. A. Quigley and A. T. Broman, "The number of people with glaucoma worldwide in 2010 and 2020," Br. J. Ophthalmol., vol. 90(3), pp. 262-267, 2006.

[11]. H. Li and O. Chutatape, "Automated feature extraction in color retinal images by a model based approach," IEEE Trans. Biomed. Eng., vol. 51, no. 2, pp. 246-254, Feb 2004.

[12]. H. Li and O. Chutatape, "Automatic location of optic disc in retinal images," in Proc. Int. 
Conf. Image Processing, vol. 2, pp. 837-840, 2001.

[13]. J. Cheng, J. Liu, D. W. K. Wong, F. Yin, C. Cheung, M. Baskaran, T. Aung, and T. Y. Wong, "Automatic optic disc segmentation with peripapillary atrophy elimination," Int. Conf. of IEEE Eng. in Med. and Bio. Soc., pp. 6624-6627, 2011.

[14]. J. Cheng, J. Liu, F. Yin, B. H. Lee, D. W. K. Wong, T. Aung, C. Y. Cheng, and T. Y. Wong, "Self-assessment for optic disc segmentation," IEEE Int. Conf. Eng. in Med. and Bio. Soc., pp. 5861-5864, 2013.

[15]. J. Cheng, J. Liu, Y. Xu, F. Yin, D. W. K. Wong, N. M. Tan, C. Y. Cheung, Y. C. Tham, and T. Y. Wong, "Superpixel classification based optic disc segmentation," In: Lee, K. M., Matsushita, Y., Rehg, J. M., Hu, Z. (eds.) ACCV, Part II, LNCS, vol. 7725, pp. 293-304, 2013.

[16]. J. Cheng, J. Liu, Y. Xu, F. Yin, D. W. K. Wong, N. M. Tan, D. Tao, Y. Cheng, T. Aung, and T. Y. Wong, "Superpixel classification based optic disc and optic cup segmentation for glaucoma screening," IEEE Trans. Med. Imaging, vol. 32, pp. 10191032, 2013.

[17]. J. Meier, R. Bock, G. Michelson, L. G. Nyl, and J. Hornegger, "Effects of preprocessing eye fundus images on appearance based glaucoma classification," Proc. CAIP, pp. 165-172, 2007.

[18]. J. Wang, J. Yang, K. Yu, F. Lv, T. Huang, and Y. Gong, "Locality-constrained linear coding for image classification," IEEE Int. Conf. on computer vision and pattern recognition, pp. 3360-3367, 2010.

[19]. J. Xu, O. Chutatape, E. Sung, C. Zheng, and P.C.T. Kuan, "Optic disk feature extraction via modified deformable model technique for glaucoma analysis," Pattern Recognition, vol. 40, pp. 2063-2076, 2007.

[20]. M. D. Abr`amoff, K. Lee, M. Niemeijer, W. L. M. Alward, E. Greenlee, K. Garvin, M. Sonka, and Y. H. Kwon, "Automated segmentation of the cup and rim from spectral domain oct of the optic nerve head," Inv Ophthalmol Vis Sci., vol. 50, pp. 57785784, 2009.

[21]. M. D. Abr`amoff, M. K. Garvin, and M. Sonka, "Retinal imaging and image analysis," IEEE Trans. Med. Imag., vol. 3, pp. 169-208, 2010.

[22]. M. D. Abr`amoff, W. L. M. Alward, E. C. Greenlee, L. Shuba, C. Y. Kim, J. H. Fingert, and Y. H. Kwon, "Automated segmentation of theoptic disc from stereo color photographs using physiologically plausible features," Invest. Ophthalmol. Vis. Sci., vol. 48, pp. 1665-1673, 2007.

[23]. M. Foracchia, E. Grisan, and A. Ruggeri, "Detection of optic disc in retinal images by means of a geometrical model of vessel structure," IEEE Trans. Med. Imag., vol. 23, no. 10, pp. 1189-1195, 2004.

[24]. N. Harizman, C. Oliveira, A. Chiang, C. Tello, M. Marmor, R. Ritch, and JM. Liebmann, "The isnt rule and differentiation of normal from glaucomatous eyes," vol. 124, pp. 1579-1583, 2006.

[25]. P. J. Foster, F. T. Oen, D. Machin, T. P. Ng, J. G. Devereux, G. J. Johnson, P. T. Khaw, and S. K. Seah, "The prevalence of glaucoma in chinese residents of singapore: a cross-sectional population survey of the tanjong pagar district," Arch. Ophthalmol., vol. 118(8), pp. 1105-1111, 2000.

[26]. R. Bock, J. Meier, G.Michelson, L. G. Nyl, and J. Hornegger, "Clas-sifying glaucoma with image-based features from fundus photographs," Proc. of DAGM, pp. 355-364, 2007.

[27]. R. Bock, J. Meier, L. G. Nyl, and G. Michelson, "Glaucoma risk index: Automated glaucoma detection from color fundus images," Med. Image Anal., vol. 14, pp. 471-481, 2010.

[28]. S. Y. Shen, T. Y. Wong, P. J. Foster, J. L. Loo, M. Rosman, S. C. Loon, W. L. Wong, S. M. Saw, and T. Aung, "The prevalence and types of glaucoma in malay people: the singapore malay eye study," Invest. Ophthalmol. Vis. Sci., vol. 49(9), pp. 38463851, 2008.

[29]. T. Damms and F. Dannheim, "Sensitivity and specificity of optic disc parameters in chronic glaucoma," Invest. Ophth. Vis. Sci., vol. 34, pp. 2246-2250, 1993.

[30]. Y. Xu, S. Lin, D. W. K. Wong, J. Liu, and D. Xu, "Efficient reconstruction-based optic cup localization for glaucoma screening," In: Mori, K., Sakuma, I., Sato, Y., Barillot, C., Nassir, N.(eds.) MICCAI 2013, Part III, LNCS, vol. 8151, pp. 445-452, 2013.

[31]. Z. Hu, M. D. Abr`amoff, Y. H. Kwon, K. Lee, and M. K. Garvin, "Automated segmentation of neural canal opening and optic cup in 3-d spectral optical coherence tomography volumes of the optic nerve head," Inv Ophthalmol Vis Sci., vol. 51, pp. 5708-5717, 2010.

[32]. Z. Zhang, B. H. Lee, J. Liu, D. W. K. Wong, N. M. TAN, J. H. Lim, F. S. Yin, W. M. Huang, and $\mathrm{H}$. Li, "Optic disc region of interest localization in fundus image for glaucoma detection in argali," Proc. of Int. 

ISSN : 2248-9622, Vol. 7, Issue 6, ( Part -7) June 2017, pp.14-18

Conf. on Industrial Electronics \&

Applications, pp. 1686-1689, 2010. 